\title{
Protein
}

\section{Misfolding Disorders}

A trip into the ER

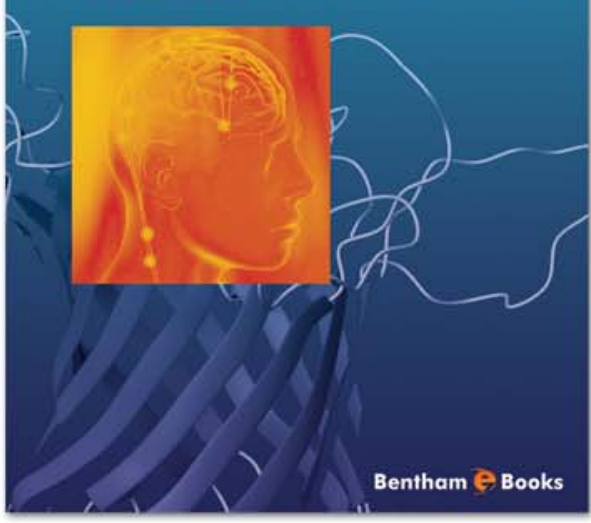

\section{Editor:}

\section{USS}

59.00

only

\section{Claudio Hetz}

Chile

\section{Protein Misfolding Disorders A Trip into the ER}

\section{wrwhenthamscience.com/ebooks/9781608050130}

\section{About the ebook}

This Ebook gives a comprehensive overview of the possible mechanisms involved in protein misfolding disorders and possible therapeutic strategies to treat these diseases. It provides the recent evidence addressing the role of cellular stress responses to neurological diseases, along with therapeutic strategies to alleviate ER stress in a disease context.

\section{Contents}

- The Unfolded Protein Response in Mammalian Cells

- The ER Chaperone GRP78 and Cancer

Endoplasmic Reticulum Stress and Protein Misfolding in Amyotrophic Lateral Sclerosis

- ER Stress Signaling Network in Pancreatic -Cells

- Heritable Neurodevelopmental Disorders and Endoplasmic Reticulum Stress

- ER Quality Control, ER Stress-Induced Apoptosis, and Neurodegenerative Diseases

- Role of Alzheimer's -Amyloid on Wnt Signaling

For Sales Advertising Inquiries: Contact: marketing@benthamscience.org 